\title{
Phase field simulations in miscibility gaps
}

\author{
M.H. Braga ${ }^{\text {a,* }}$, J.C.R.E. Oliveira ${ }^{\text {b }}$, L.F. Malheiros ${ }^{c}$, J.A. Ferreira ${ }^{d}$ \\ ${ }^{a}$ CEMUC, Department of Eng. Physics, FEUP, R. Dr. Roberto Frias s/n, 4200-465 Porto, Portugal \\ ${ }^{\mathrm{b}}$ CFP, Department of Eng. Physics, FEUP, R. Dr. Roberto Frias s/n, 4200-465 Porto, Portugal

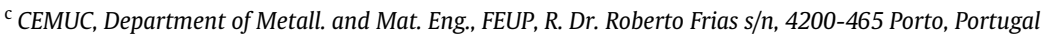 \\ d LNEG Laboratory, R. da Amieira - Apartado 1089, S. Mamede Infesta, Portugal
}

\section{A R T I C L E I N F O}

\section{Article history:}

Received 19 May 2008

Received in revised form 29 September 2008

Accepted 6 October 2008

Available online 6 November 2008

\section{Keywords:}

Spinodal

Phase field simulations

$\mathrm{Bi}-\mathrm{Zn}$

Vycor

Sol-gel

\section{Introduction}

There are many systems that present miscibility gaps in the liquid or solid phase. These systems are, for example, Bi-Zn, Li-Zr, $\mathrm{Mg}-\mathrm{Mn}, \mathrm{S}-\mathrm{Sb}, \mathrm{Sn}-\mathrm{P}, \mathrm{Ti}-\mathrm{W}, \mathrm{Cu}-\mathrm{Ni}-\mathrm{Sn}$ or even glasses, such as Vycor $^{\circledR}$, that contains approximately $75 \mathrm{wt} \% \mathrm{SiO}_{2}, 20 \mathrm{wt} \% \mathrm{~B}_{2} \mathrm{O}_{3}$ and $5 \mathrm{wt} \% \mathrm{Na}_{2} \mathrm{O}$.

In the ternary phase diagram of $\mathrm{B}_{2} \mathrm{O}_{3}-\mathrm{SiO}_{2}-\mathrm{Na}_{2} \mathrm{O}$, Vycor ${ }^{\circledR}$ corresponds to a composition in which at a given temperature, two immiscible liquid phases are formed, one of them rich in $\mathrm{SiO}_{2}$. When the sample is quenched from the miscibility gap, the two phases corresponding to the immiscible liquids are kept. For industry and for most of the applications only the $\mathrm{SiO}_{2}$ rich phase is important, and so the other phase will be leach out leaving a porous, high-silica skeleton [1].

In this paper, the dynamics of a thermodynamically unstable solution with respect to composition variations is studied. In such a regime, nucleation of the new phase is not necessary. The phase transformation occurs spontaneously by spinodal decomposition

\footnotetext{
* Corresponding author. Tel.: +351 225081420; fax: +351225081447

E-mail addresses: mbraga@fe.up.pt (M.H. Braga), jespain@fe.up.pt (J.C.R.E. Oliveira), lfmf@fe.up.pt (L.F. Malheiros), Jorge.Ferreira@ineti.pt (J.A. Ferreira).
}

and may result in a multi-phase microstructure in which phases are highly interconnected (for a certain range of compositions and temperatures of the spinodal region). The latter microstructures have numerous applications: one of them already mentioned is Vycor $^{\circledR}$ glass, whose silica structure can be the matrix for other applications such as the study of superfluids [2].

Another very important and up-to-date application concerns the sol-gels for the production of nanoparticles [3] and membranes, with applications in health and in food technology [4].

The spinodal decomposition may also be used to improve the mechanical properties of certain materials since, in general, spinodally decomposed materials can exhibit very fine scale composition modulations, resulting in very high strength materials. The phase field simulations of solid or liquid miscibility gaps may be used to determine how the mechanical properties (local stress, strain fields or Young's modulus) depend on the composition of the blend. For example, $\mathrm{Cu}-\mathrm{Ni}-\mathrm{Sn}$ alloys can be hardened by spinodal transformation and are used in electrical contact materials that grip by elastic springback, such as in computer connectors [5].

Lead free solder materials are under investigation for environmental reasons. Structural and mechanical properties are also of great importance in what concerns solders. In order to study the mechanical properties of amorphous solders alloys, it is crucial to study the liquid phase.

The phase field method is a subject of interest since a long time ago. Based on the Ginzburg-Landau theory of phase 


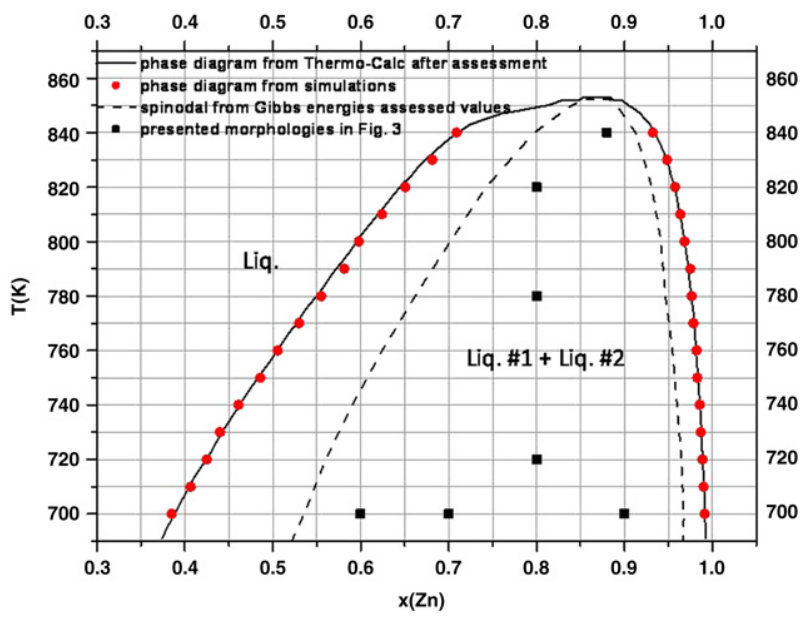

Fig. 1. The zoom of the miscibility gap occurring in the liquid region as well as its spinodal region obtained from Gibbs energy by [16] after [14]. The scale limits, which are the values of $\phi$ for each temperature obtained with the simulations, are represented by the two opposing circles in the phase diagram miscibility gap curve $(x$ (Liquid\# $1, \mathrm{Zn})$ and $x$ (liquid\#2, $\mathrm{Zn})$ ); points in squares present variations of the simulated asymptotic morphology at different temperatures and compositions shown in Fig. 3.

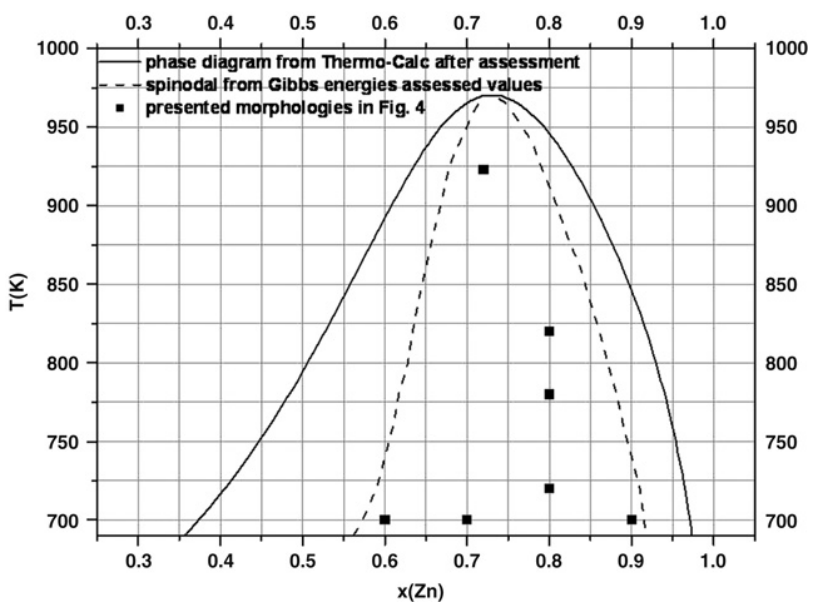

Fig. 2. The zoom of the miscibility gap occurring in the liquid region as well as its spinodal region obtained from Gibbs energy by [15]; points in squares present variations of the simulated asymptotic morphology at different temperatures and compositions shown in Fig. 4.

transitions, one of the first applications of phase field method was developed by Cahn-Hilliard [6] which describes the process of phase separation, by which the two components of a binary solution spontaneously separate. The main feature of this method is to substitute boundary conditions at the interface by a partial differential equation for the evolution of an auxiliary field (the phase field) that takes the role of an order parameter. Since the seventies [7] and mostly on the nineties, the multiphase field concept has been developed by [8-13].

Using phase field simulations, it is possible to simulate the dynamics of immiscible liquids appearing at the miscibility gap of an alloy, just by having access to the Gibbs energy of the liquid phase for each composition and temperature. These simulations may be used to determine the asymptotic compositions of the fluids for a given Gibbs energy. Even more, it is known that different parameters of the excess Gibbs energy of a certain phase may exhibit different asymptotic morphologies, in spite of the similarity of the associated equilibrium curves. Hence, these morphological studies may be an efficient method to distinguish between different Gibbs energies that give approximately the same equilibrium compositions, in spite of having different corresponding parameters of the excess Gibbs energy.

In this work we have performed phase field simulations for the two liquids separation occurring in the miscibility gap of the $\mathrm{Bi}-\mathrm{Zn}$ system, for different temperatures and concentrations. We have used the $\mathrm{Bi}-\mathrm{Zn}$ Gibbs energy previously calculated using the CALPHAD method and found a rich diversity of asymptotic morphologies for different points of the $\mathrm{Bi}-\mathrm{Zn}$ phase diagram. It was also possible to determine the asymptotic compositions of Liq.\#1 and Liq.\#2. The latter calculations were performed for two different Gibbs energies representing the liquid phase of the $\mathrm{Bi}-\mathrm{Zn}$ system obtained by two different authors [14,15] using the CALPHAD method.

\section{Theory and calculation}

\subsection{Determination of the Gibbs energies using the CALPHAD method}

The CALPHAD method was used prior to this work to obtain the Gibbs energy of the liquid phase [16].

The method for the calculation of thermodynamic equilibrium is based on modeling the Gibbs energies of all coexisting phases and minimizing the total Gibbs energy of the system. The system $\mathrm{Bi}-\mathrm{Zn}$ was assessed by Malakhov [14] but some changes were introduced in the first assessment by [16].

The Gibbs energy of mixing of the substitutional solution (liquid phase mixture in this case) is expressed by [17], as:

$G_{\text {mix }}=G^{i d}+G^{E}$

where $G^{i d}=R T \sum_{i} x_{i} \ln \left(x_{i}\right)$ is the ideal mixing contribution, $x_{i}$ is the molar fraction of the component $i$ with $\sum_{i} x_{i}=1$ and $G^{E}=\sum_{i} \sum_{i>j} x_{i} x_{j} L_{i j}$ (with $L_{i j}=\sum_{v=0}^{k}\left(x_{i}-x_{j}\right)^{v} \cdot{ }^{v} L_{i j}$ where $\left.{ }^{v} L_{i j}=a_{v}+b_{v}+c_{v} T \ln T\right)$ the contribution due to non-ideal interactions between the components (also known as the excess Gibbs energy of mixing) where $x_{i}$ and $x_{j}$ are the molar fractions of the components $i$ and $j\left(x_{j}=1-x_{i}\right)$.

In this work we have used the assessments of two different authors: Vizdal et al. [16], after Malakhov [14], and Djaballah et al. [15].

Vizdal et al. [16], after Malakhov [14], assessed the liquid phase using the excess Gibbs energy written as:

$$
\begin{aligned}
& G^{E}=x_{\mathrm{Bi}} x_{\mathrm{Zn}}\left[{ }^{0} L_{\mathrm{Bi}, \mathrm{Zn}}+\left(x_{\mathrm{Zn}}-x_{\mathrm{Bi}}\right) \cdot{ }^{1} L_{\mathrm{Bi}, \mathrm{Zn}}+\left(x_{\mathrm{Zn}}-x_{\mathrm{Bi}}\right)^{2} \cdot{ }^{2} L_{\mathrm{Bi}, \mathrm{Zn}}\right. \\
& \left.+\cdots+\left(x_{\mathrm{Zn}}-x_{\mathrm{Bi}}\right)^{6} \cdot{ }^{6} L_{\mathrm{Bi}, \mathrm{Zn}}\right]
\end{aligned}
$$

with ${ }^{v} L_{\mathrm{Bi}, \mathrm{Zn}}=a_{v}+b_{v} T$ ( $a$ and $b$ where determined using the CALPHAD method and experimental data).

Djaballah et al. [15] assessed the liquid phase using the excess Gibbs energy written as follows:

$$
\begin{aligned}
& G^{E}=x_{\mathrm{Bi}} x_{\mathrm{Zn}}\left[{ }^{0} L_{\mathrm{Bi}, Z \mathrm{Zn}}+\left(x_{\mathrm{Zn}}-x_{\mathrm{Bi}}\right) \cdot{ }^{1} L_{\mathrm{Bi}, Z \mathrm{n}}+\left(x_{\mathrm{Zn}}-x_{\mathrm{Bi}}\right)^{2} \cdot{ }^{2} L_{\mathrm{Bi}, \mathrm{Zn}}\right. \\
& \left.+\left(x_{\mathrm{Zn}}-x_{\mathrm{Bi}}\right)^{3} \cdot{ }^{3} L_{\mathrm{Bi}, Z \mathrm{n}}\right]
\end{aligned}
$$

with ${ }^{v} L_{\mathrm{Bi}, \mathrm{Zn}}=a_{v}+b_{v} T+c_{v} \ln T$ ( $a, b$ and c where determined using the CALPHAD method and experimental data).

\section{Phase field simulations}

During the phase separation occurring at the spinodal region of Bi-Zn system, a mixture of Liq.\#1 and Liq\#2 is formed. Because the mixture is incompressible we can characterize the system by one order parameter, $\phi$, which is defined as $\phi=x_{\mathrm{Zn}}-x_{\mathrm{Bi}}$. The free energy of the system can be written, phenomenologically, as the Landau functional $[6,18]$,

$F[\phi]=\int \mathrm{d} \vec{r}\left[k(\nabla \phi)^{2}+G^{\text {liquid }}(\phi)\right]$, 


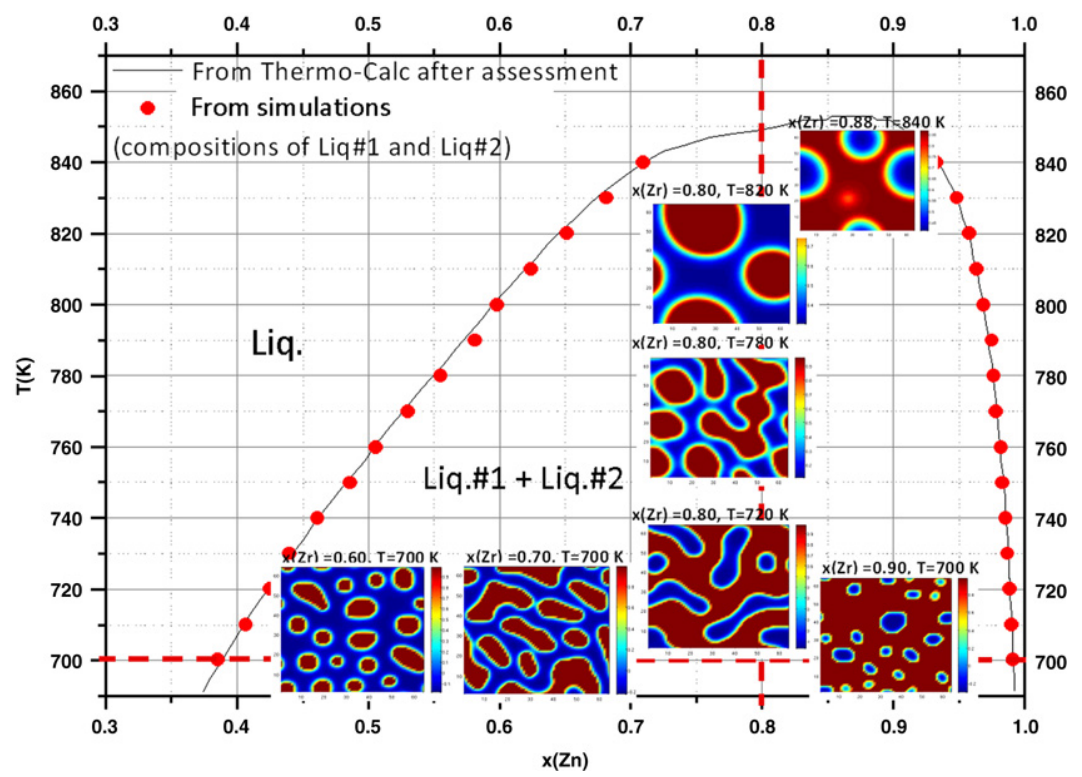

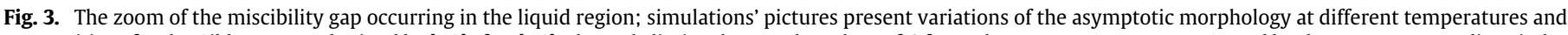

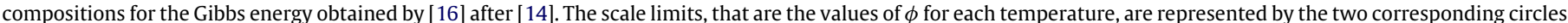

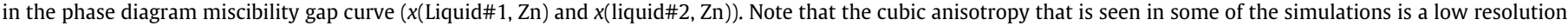
effect with no physical meaning.

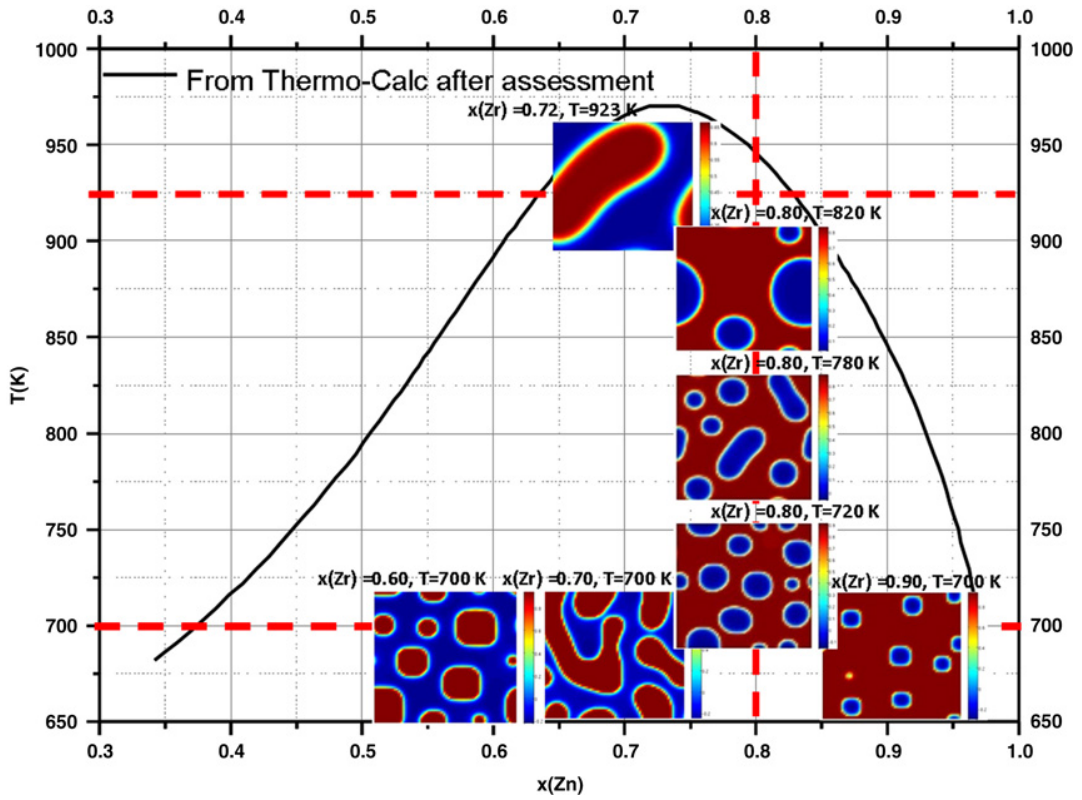

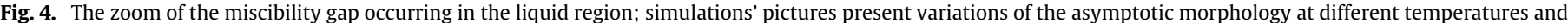

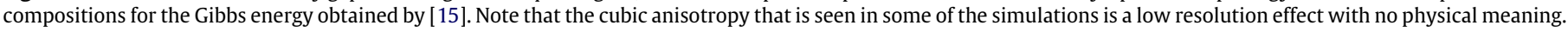

where $G^{\text {liquid }}(\phi)$ is the Gibbs energy $G_{\text {mix }}$, presented in (1) (making $\phi=x_{\mathrm{Zn}}-x_{\mathrm{Bi}}$ ). The surface tension corresponds to the energy of the flat interface per unit length and is given by $\sigma=k \int \mathrm{d} n\left(\frac{\partial \phi}{\partial n}\right)^{2}$, where the integral is performed across the interface and in the direction normal to it. The functional derivative of $F$ is $\delta F / \delta \phi=$ $k \nabla^{2} \phi-\frac{\partial G^{\text {liquid }}}{\partial \phi}$.

The time evolution of the order parameter can be described by Cahn-Hilliard equation for the phase separation in isotropic solids or liquids at the diffusive regime $[6,18]$, which can be written in the form of a continuity equation $\partial \phi / \partial t=-\nabla \vec{j}$, with current $\vec{j}=-\nabla(\delta F / \delta \phi)$. Since $\phi$ is conserved in the total volume, $\partial \phi / \partial t$ is the divergence of a flux $\vec{j}$,

$\frac{\partial \phi}{\partial t}=-\nabla \cdot \vec{j}=\nabla^{2}\left(\frac{\partial G^{\text {liquid }}}{\partial \phi}-k \nabla^{2} \phi\right)$.

The asymptotic growth regime for phase separation in binary liquids, in the diffuse regime, is given by $L \propto t^{1 / 3}$, where $L$ is the characteristic length of the phase boundaries. Thus, the asymptotic evolution is very slow leading to almost stationary morphologies for large evolution times. The time that is needed to reach the asymptotic growth regime in a given sample will in general depend on the mobility of the alloy species. In a future work we intend to calibrate the key parameters characterizing the 

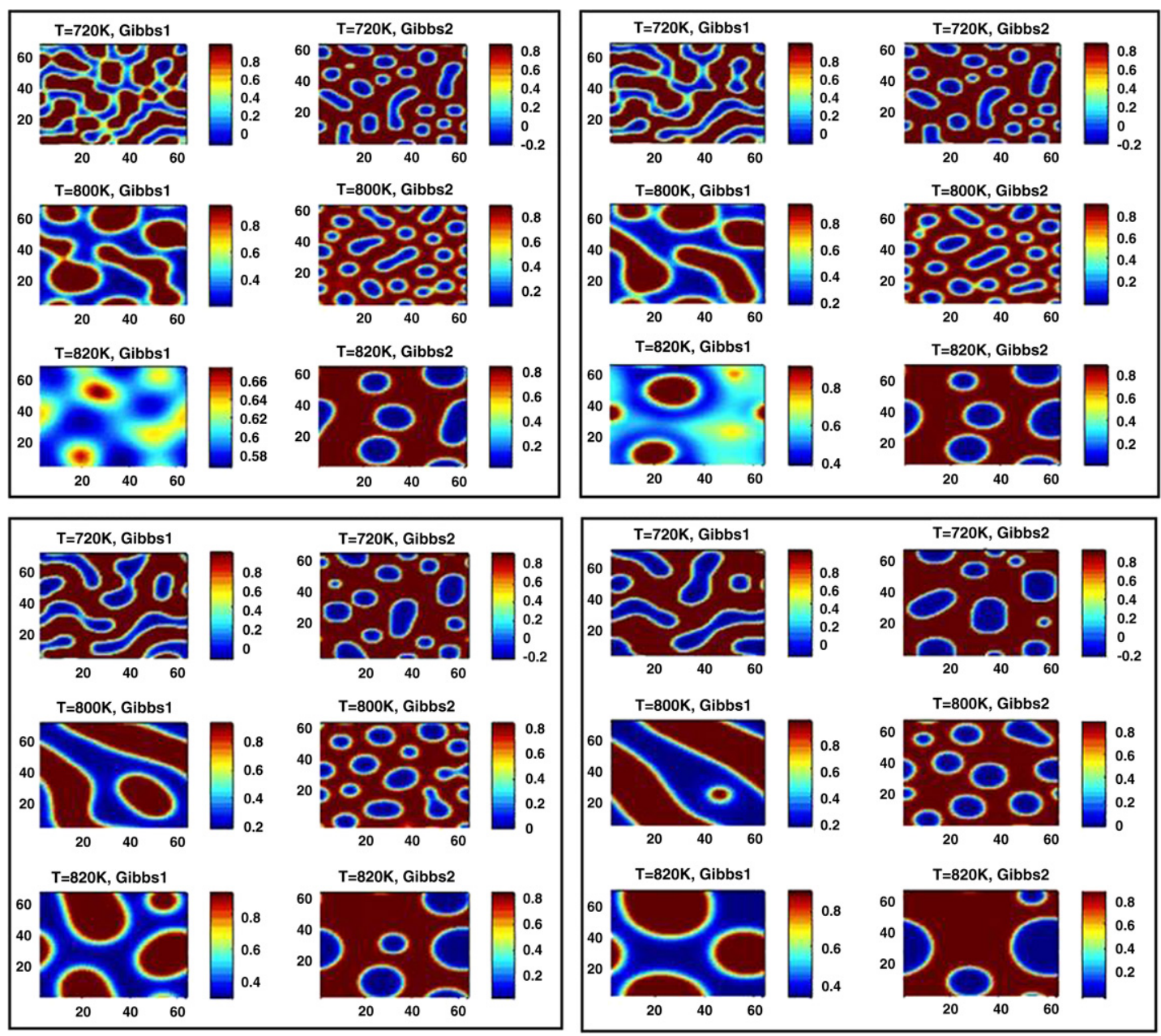

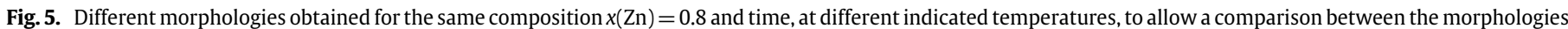

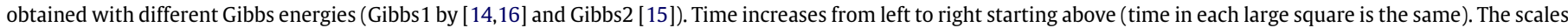
correspond to the values of $\phi$.

system by performing a detailed quantitative study of the time evolution of $L$ in the simulations and a subsequent comparison with the observed one in different system samples.

In Eq. (3) changing the time scale can scale out $k$. In fact the same dynamics can be obtained with a different surface tension by changing the size of the simulation box accordingly. Hence, the asymptotic morphology of the system is just a function of the Gibbs energy and not of the value of $k$.

In the alloy system, the atoms of $\mathrm{A}$ and $\mathrm{B}$ ( $\mathrm{Bi}$ and $\mathrm{Zn}$ in this case) can exchange position only locally (not over large distances), leading then to a diffusive transport of the order parameter.

We have integrated (3) using a standard finite-difference method [19]. In all simulations we have used initial random conditions for $\phi$ and $k=1$.

\section{Results and discussion}

In Figs. 1 and 2 both the miscibility gap and spinodal curves, obtained after the assessments of [14,16] and [15] respectively, can be observed. In Fig. 1 the points corresponding to the asymptotic values of $x$ (Liq.\#1, Zn) and $x$ (Liq.\#2, Zn), obtained in this work by phase field simulations, can also be observed.

In Fig. 3, the miscibility gap curve of the Bi-Zn system is shown. A good agreement, between the equilibrium curve calculated in [16] and the one obtained by the simulations was found as expected. Here, the simulations' images for different compositions and temperatures represent the asymptotic morphologies of the $\mathrm{Bi}-\mathrm{Zn}$ system near the equilibrium configuration. It can be seen that the size and shape of the domains change considerably with temperature for the same concentration, and with the concentration, for the same temperature.

In Fig. 4 equivalent results to those of Fig. 3 are shown. It can be observed that the morphologies corresponding to the same temperatures and compositions are considerably different. For instance, interconnected structures may appear in a region of the miscibility gap of Fig. 4 where in Fig. 3 round shaped domains appear.

By the analysis of the asymptotic morphologies shown inside the miscibility gap, it can be seen that for the compositions near each side of the spinodal line (Figs. 1-4), there is a matrix of the more abundant liquid and inside this matrix, isolated round shaped domains of the other liquid phase appear. Concerning the system' compositions that are more in the middle of the spinodal region, for the same temperature as the previously referred morphologies, interconnected domains can be observed. These interconnected domains have many applications and the identification of the compositions for which they appear is crucial $[20,21]$.

Note for example that, for $x(\mathrm{Zn})=0.8$ in Fig. 3, interconnected domains appear at least for $T \leq 780 \mathrm{~K}$. Only round shaped domains can be observed for $x(\mathrm{Zn})=0.8$ in Fig. 4 . If the phase diagrams and spinodal lines are compared, it can be seen that the latter composition in the miscibility gap of Fig. 1 corresponds to the center of the spinodal region and that at $T=720 \mathrm{~K}$ it is clear 

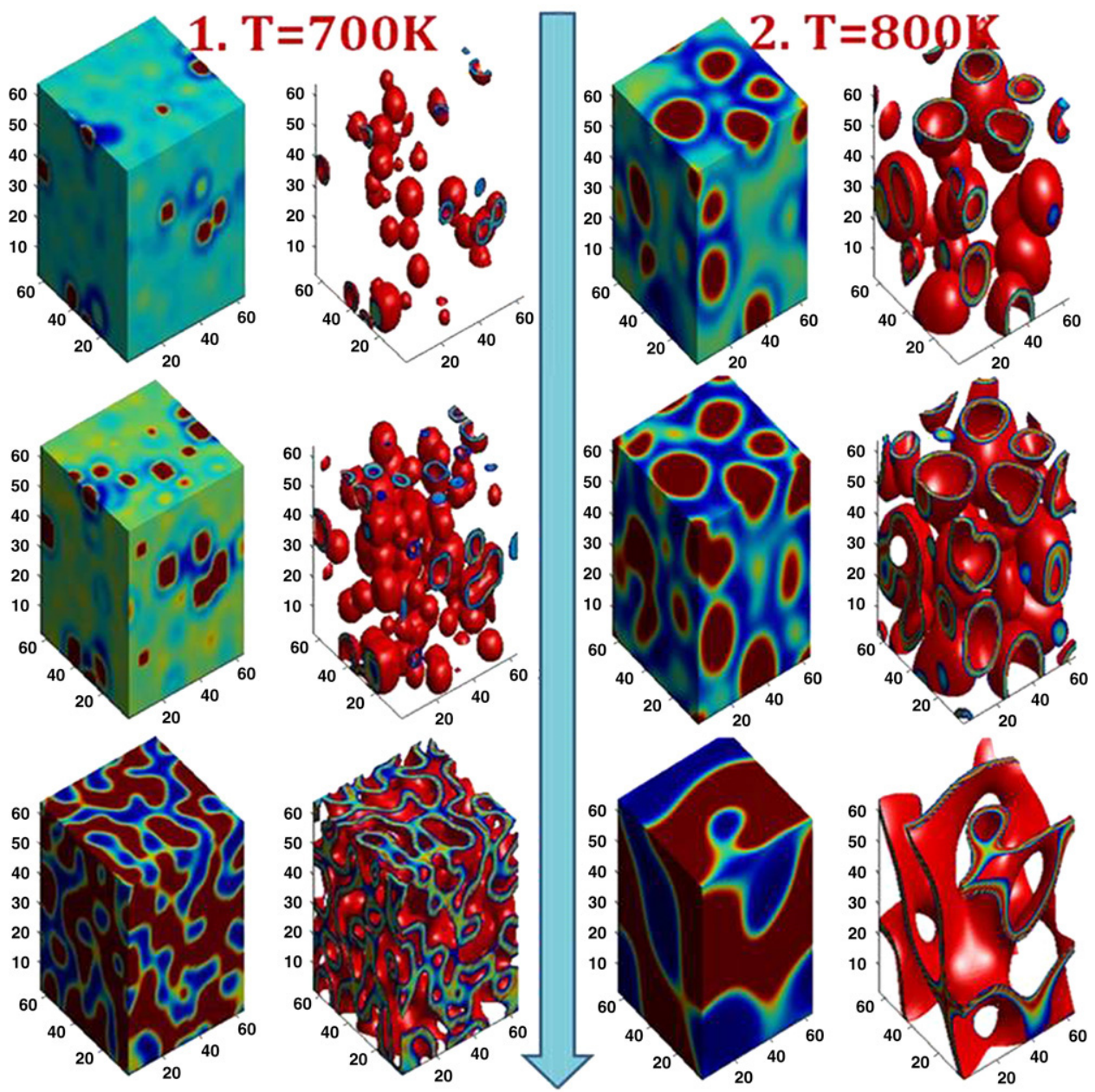

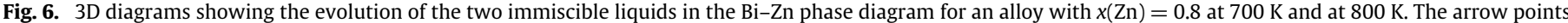
the direction of time evolution. The Gibbs energy used was determined by $[14,16]$.
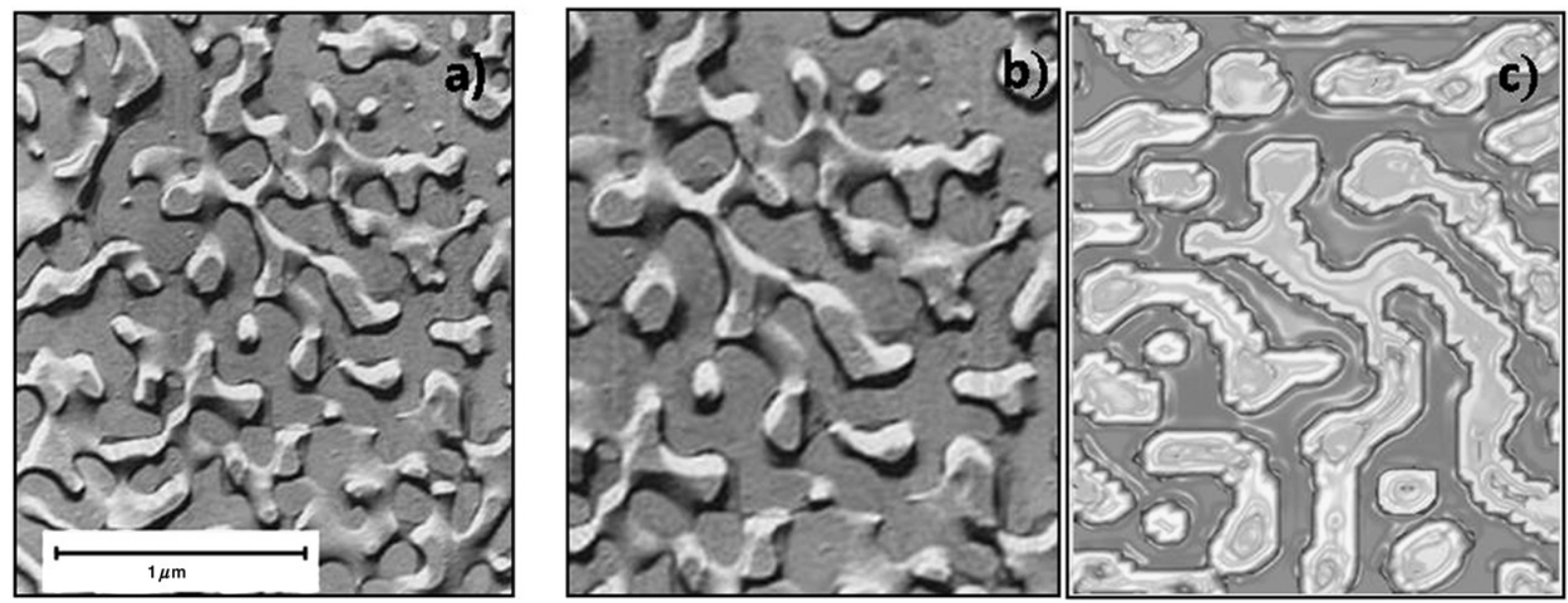

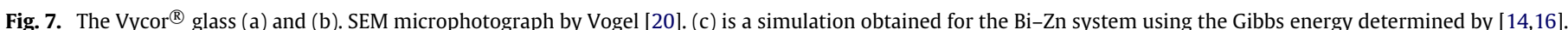

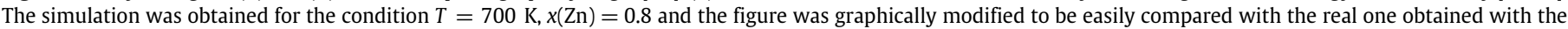

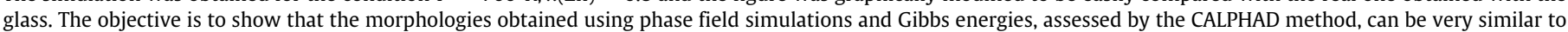
the real ones for different systems.

that Liq.\#1 is more abundant (matrix is Liq.\#1). On the contrary, by the observation of Fig. 2 , it can be seen that $x(\mathrm{Zn})=0.8$ does not correspond to the center of the spinodal and that at $T=720 \mathrm{~K}$ it is clear that Liq.\#2 is more abundant (matrix is Liq.\#2).
The analysis of the morphologies as a function of temperature also reveals that the interconnected domains will change to spheres as the temperature rises to the upper limit of the miscibility gap. 

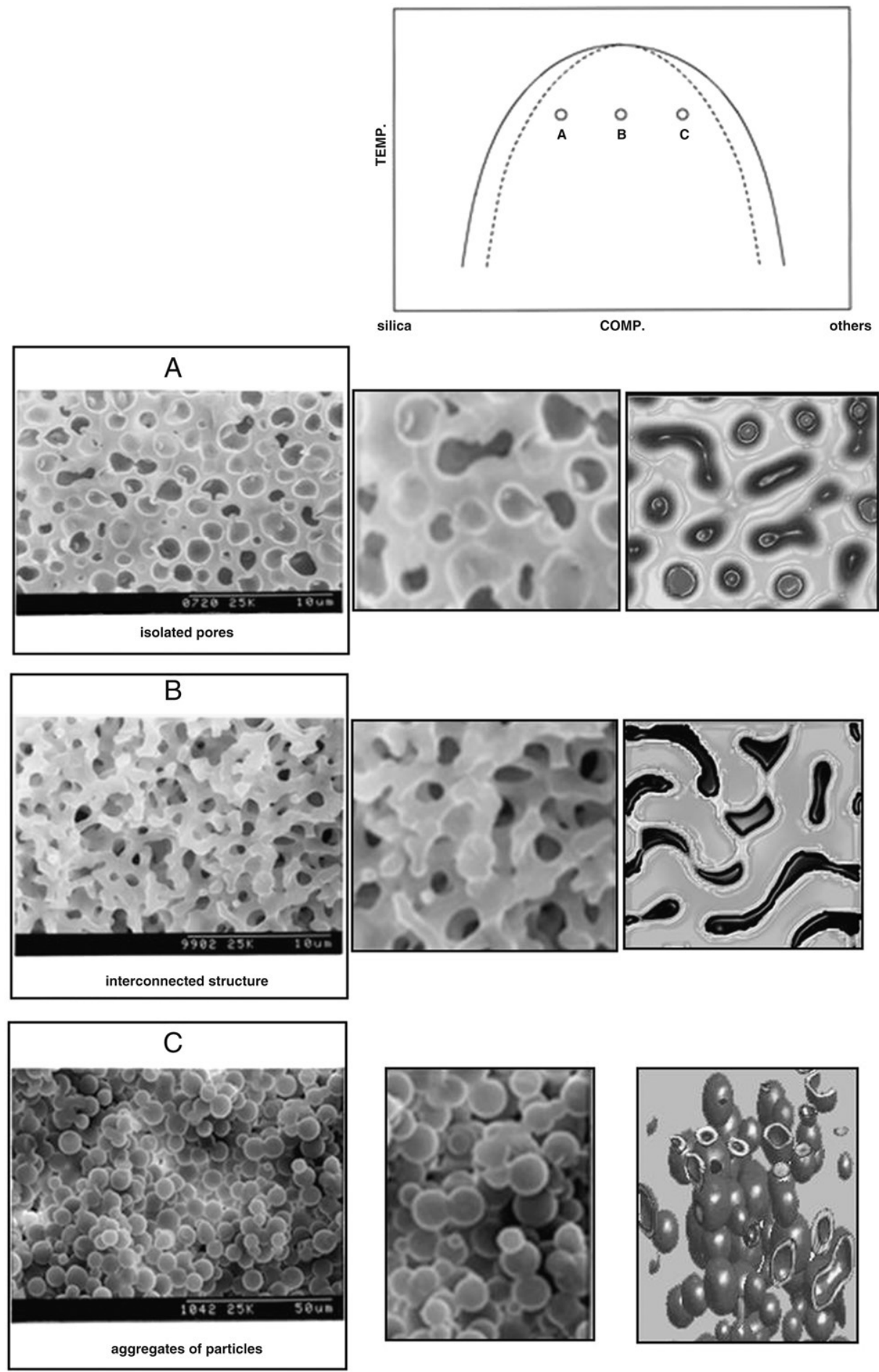

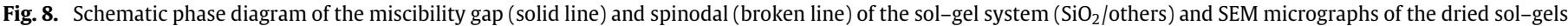

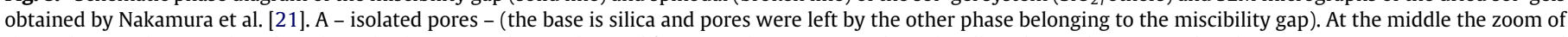

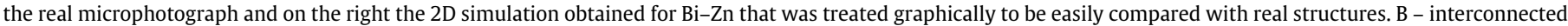

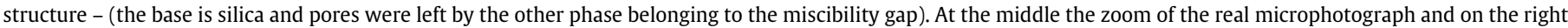

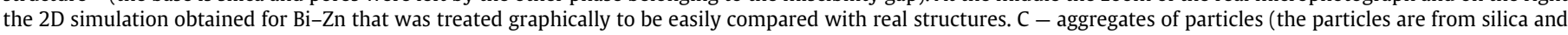

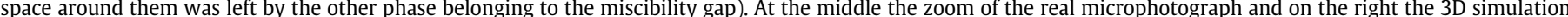

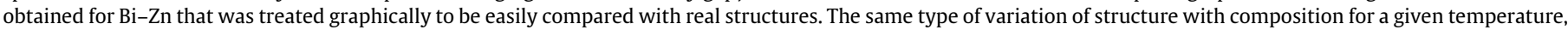
that can be seen in A, B, and C, can also be observed on the simulations of the Bi-Zn (Figs. 3 and 4) at T=700 K.

The same type of composition and temperature morphologies dependence was found in [20,21].

It can also be seen in Fig. 5 that the dynamics toward equilibrium also differs when different Gibbs energies are considered. We have performed 3D simulations of the phase separation for the
Gibbs energy of $[14,16]$, as it can be observed in Fig. 6, where the evolution of the phase boundaries is presented for two different temperatures.

Although Vycor and sol-gel cannot be directly compared with $\mathrm{Bi}-\mathrm{Zn}$, it is relevant to show that, in spite of their different Gibbs 
energies, similar morphologies can be observed in corresponding relative positions of the miscibility gap and spinodal regions. This fact enhances the possible applications of the method.

Figs. 7 and 8 show comparisons between our Bi-Zn simulated morphologies (obtained with the Gibbs energy [14,16]) and similar ones for observed samples of Vycor $^{\circledR}$ [20] and sol-gel [21], respectively.

\section{Conclusions}

Using phase field simulations, the dynamics of the two immiscible liquids appearing on the phase diagram of the $\mathrm{Bi}-\mathrm{Zn}$ system was studied. Good agreement between the miscibility gap curve determined by the simulations and the one obtained by the CALPHAD method was found, as expected.

It was found a rich diversity of asymptotic morphologies for different points of the $\mathrm{Bi}-\mathrm{Zn}$ phase diagram.

We have compared the morphologies for two different Gibbs energies determined by Calphad method and found very different morphologies for the same times, concentrations and temperatures. Thus, it can be concluded that the different morphologies and the time it takes to reach them is a signature of the calculated excess Gibbs energy parameters for a given system.

It could also be verified the similarities between the simulated structures and those observed in different real systems, such as Vycor ${ }^{\circledR}$ glass and sol-gel.

It can be pointed out, that for a given temperature, the morphologies of the $\mathrm{Bi}-\mathrm{Zn}$ system depend on the composition in a similar way as those observed in the sol-gel system, especially the morphologies obtained with one of the Gibbs energies.

The results obtained in this work show that using the Gibbs energies, obtained for example with the CALPHAD method and phase field simulations, we have a straightforward method to determine the morphologies of the miscibility gap as a function of the concentration, temperature and time. This method may be useful to find the best structures, depending on the applications and purposes; for instance, for the fabrication of nanoparticles.

\section{Acknowledgements}

The authors would like to acknowledge the COST MP0602 action: "Advanced Solder Materials for High-Temperature Application - their nature, design, process and control in a multiscale domain".

\section{References}

[1] M.H. Bartl, K. Gatterer, H.P. Fritzer, S. Arafa, Spectrochim. Acta A 57 (2001) 1991-1999.

[2] B. Peter, B. Paul, L. Gavin, C. Lee, M. Akira, I. Osamu, M. Pinaki, Phys. Lett. A 310 (4) (2003) 311-321.

[3] A. Bouchara, G. Mosser, G.J.A.A. Soler-Illia, J.-Y. Chane-Ching, C. Sanchez, J. Mater. Chem. 14 (2004) 2347-2354.

[4] P. Schurtenberger, Nanotech 2005, California, U.S.A., May 8-12, 2005.

[5] S.M. Allen, E.L. Thomas, The Structure of Materials, Wiley MIT, 1999, pp. 374.

[6] J.W. Cahn, J.E. Hilliard, J. Chem. Phys. 28 (2) (1958) 258.

[7] J.S. Langer, H. Müller-Krumbhaar, Acta Metall. 26 (1978) 1681, 1689, 1697.

[8] L.Q. Chen, A.G. Khachaturyan, Acta Metall. Mater 39 (11) (1991).

[9] A.A. Wheeler, W.J. Boettinger, G.B. Mc Fadden, Phys. Rev. A 45 (10) (1992) 7424

[10] G. Caginalp, E. Socolovsky, SIAM J. Sci. Comput. 15 (1) (1994) 106

[11] R. Kobayashi, Physica D 63 (3,4) (1993) 410.

[12] A.A. Wheeler, B.T. Murray, R.J. Schaefer, Physica D 66 (1,2) (1993) 243.

[13] J. Steinbach, F. Pezzolla, B. Nestler, M. Seeßelberg, R. Prieler, G.J. Schmitz, J.L.L. Rezende, Physica D 94 (1996) 135.

[14] D.V. Malakhov, CALPHAD 24 (2000) 1-14.

[15] Y. Djaballah, L. Bennour, F. Bouharkat, A. Belgacem-Bouzida, Modeling. Simul. Mater. Sci. Eng. 13 (2005) 361-369.

[16] J. Vizdal, M.H. Braga, A. Kroupa, K.W. Richter, D. Soares, L.F. Malheiros, J. Ferreira, CALPHAD 31 (2007) 438-448.

[17] O. Redlich, A. Kister, Ind. Eng. Chem. 40 (1948) 411-420.

[18] A.J. Bray, Adv. Phys. 43 (1994) 357-458.

[19] J.C.R.E. Oliveira, C.J.A.P. Martins, P.P. Avelino, Phys. Rev. D 71 (2005) 083509.

[20] W. Vogel, Chemistry of the Glass, The American Ceramics Society, 1985, pp. 83.

[21] N. Nakamura, R. Takahashi, S. Sato, T. Sodesawa, S. Yoshida, Phys. Chem. Chem. Phys. 2 (2000) 4983-4990. 